\title{
Erratum to: A new ozone denuder for aerosol sampling based on an ionic liquid coating
}

\author{
Alexandre Albinet $\cdot$ Nicolas Papaiconomou • \\ Julien Estager • Joël Suptil • Micheline Draye • \\ Jean-Luc Besombes
}

Published online: 21 March 2010

(C) Springer-Verlag 2010

\section{Erratum to: Anal Bioanal Chem}

\section{DOI 10.1007/s00216-009-3243-5}

Regrettably, Micheline Draye was missing as co-author in the final manuscript submitted for publication by the authors.

The correct authors of this article are

Alexandre Albinet, Nicolas Papaiconomou, Julien Estager, Joël Suptil, Micheline Draye and Jean-Luc Besombes org/10.1007/s00216-009-3243-5.

\footnotetext{
A. Albinet $(\bowtie) \cdot N$. Papaiconomou $(\bowtie) \cdot J$. Estager $\cdot$ J. Suptil $\cdot$ M. Draye $\cdot$ J.-L. Besombes Laboratoire Chimie Moléculaire Environnement (LCME-Polytech'Savoie), Université de Savoie, Campus de Savoie Technolac,

Le Bourget du Lac 73376, France e-mail: alexandre.albinet@gmail.com

e-mail: nicolas.papaiconomou@univ-savoie.fr
} 